\title{
Study and Optimization of Raman Amplifiers in Tellurite-Based Optical Fibers for Wide- Band Telecommunication Systems
}

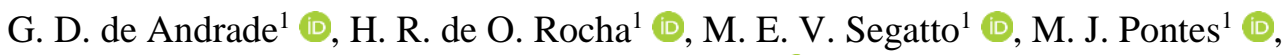 \\ C. E. S. Castellani ${ }^{1}(\mathbb{D}$ \\ ${ }^{1}$ Federal University of Espirito Santo (UFES), Electrical Engineering Department, Av. Fernando Ferrari, \\ Vitoria-ES, 29075-073, carlos.castellani@ufes.br
}

\begin{abstract}
Here we present a numerical analysis and the optimization of a multi-pump discrete Raman amplification system based on the use of a Tellurite optical fiber as the gain media. By using previously reported optimization techniques we were able to exploit the high Raman gain of Tellurite optical fibers and its multipeak spectrum to achieve amplification over an amplification band from 1520 to $1600 \mathrm{~nm}$. Average gains around $10 \mathrm{~dB}$ with ripples in the region of $3 \mathrm{~dB}$ were achieved using only 3 pumps with less than $800 \mathrm{~mW}$ each. Optimized systems were obtained for different lengths of fiber showing that discrete Raman amplifiers based on tellurite fibers with lengths as short as $\mathbf{1 0 0}$ meters can provide high gains and low ripples in a broad wavelength band. Lower cost systems using only 2 pumps were also analyzed in this study providing $5.8 \mathrm{~dB}$ of gain with only $2.4 \mathrm{~dB}$ of ripple for an $80 \mathrm{~nm}$ signal bandwidth.
\end{abstract}

Index Terms - Non-linear optics, Raman amplifiers, Raman Scattering, Tellurite fibers.

\section{INTRODUCTION}

The development in the last decades of tellurite $\left(\mathrm{TeO}_{2}\right.$-based) optical fiber technology has found use in various applications as optical non-linear devices in both near and mid-infrared (IR) region [1]. The fact that its transparency window can extend up to 5-6 $\mu \mathrm{m}$ [2], still presenting high levels of nonlinearities makes this type of glasses ideal hosts, for example, for the generation of supercontinuum [3], optical parametric amplification [4] and Raman lasers [5] in regions of the spectrum that cannot be covered by silica fibers. Moreover, tellurite-based glasses present high rare earth ions (RE) solubility, allowing them to be used as active devices emitting light at various wavelengths when doped with elements such as Ytterbium $\left(\mathrm{Yb}^{+3}\right)$, Thulium $\left(\mathrm{Tm}^{+3}\right)$ and Holmium $\left(\mathrm{Ho}^{+3}\right)$ [6].

Another important aspect of tellurite-based fibers is that, apart from the high levels of nonlinearities, they present Raman gains that are usually 30 to 50 times that of silica fibers [2],[7], which can be further extended by tailoring such glasses in PCF geometries [4],[8] or by combining them with germanate [9] or fluorite glasses [10]. This feature has already been explored in a number of numerical studies [5] and experimental demonstrations of Raman shifters [11] and lasers [12] at various regions of the spectrum. Nevertheless, the amount of research dedicated to analyze the use of tellurite-based fibers as the Raman gain medium for telecommunication systems has been very modest 


\section{[13]-[15].}

Broadband amplification in tellurite-based fibers has already been achieved for wavelength ranges up to $160 \mathrm{~nm}$ [14],[15]. Even though high average gains higher than $10 \mathrm{~dB}$ were obtained in those systems, no special care was taken into significantly reducing the values of ripple, which were of the order of $10 \mathrm{~dB}$ in [14] and more than $20 \mathrm{~dB}$ in [15]. This issue was addressed in [13] where a ripple as low as $1 \mathrm{~dB}$ could be achieved for a wavelength band of $160 \mathrm{~nm}$. However, this low value of ripple was obtained at the expense of using a significantly more complex pumping system with 8 pumps.

Here we present an optimization of multi-pumped $80 \mathrm{~nm}$ broad Raman amplifiers based on tellurite fibers, more precisely a $\mathrm{TeO}_{2}-\mathrm{Bi}_{2} \mathrm{O}_{3}-\mathrm{ZnO}-\mathrm{Na}_{2} \mathrm{O}(\mathrm{TBZN})$ glass-based fiber, that allowed achieving high levels of gains maintaining low levels of ripple ( $2 \mathrm{~dB})$, using only simple configurations with 2 or 3 low-power pumps in order to reduce complexity and costs. The amplifiers were optimized using very fast and simple analytic and numerical techniques, which allowed also for an optimization of the length of the fiber to be used as the gain medium. In order to evidence the benefits of the use of tellurite-based fibers as discrete Raman amplifiers a comparison with optimized silica-based systems using the same techniques is performed. The results obtained here highlight the potential of using such glasses as host for short-length and high-gain broadband Raman amplification in telecommunication systems.

\section{SETUP AND OPTIMIZATION TECHNIQUES}

The setup utilized in our simulations is shown in Fig. 1. We use 20 equally spaced signals in the wavelength range from 1520 to $1600 \mathrm{~nm}$ with $-5 \mathrm{dBm}$ each, which are coupled into a tellurite-based fiber that is counter-pumped by 2 or 3 pumps. The telluride fiber used in our simulations is based in the fiber experimentally demonstrated in [15], which is made of a $\mathrm{TeO}_{2}-\mathrm{Bi}_{2} \mathrm{O}_{3}-\mathrm{ZnO}-\mathrm{Na}_{2} \mathrm{O}$ (TBZN) glass, from here onward called simply as tellurite fiber. Its Raman gain curve is shown in Fig.2 where a gain peak of $55 \mathrm{~W}^{-1} \cdot \mathrm{km}^{-1}$ is obtained for a wavelength shift of $22.2 \mathrm{THz}$. As it is commonly seen in the gain curve of tellurite fibers it presents a second peak at a wavelength shift of around $13 \mathrm{THz}$ with a gain peak of $38.4 \mathrm{~W}^{-1} \cdot \mathrm{km}^{-1}$, which is still an order of magnitude higher than the gain obtained with standard silica fibers.

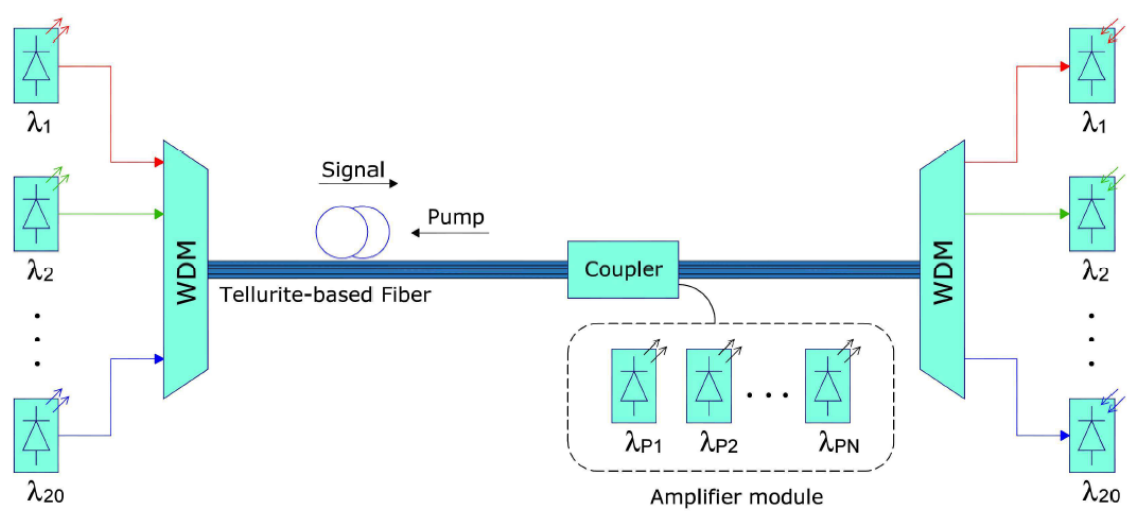

Fig. 1. Schematic of the optimized multi-pump Raman amplification system.

Brazilian Microwave and Optoelectronics Society-SBMO received 30 Nov 2018; for review 6 Dec 2018; accepted 18 Apr 2019 
The high-gain two-peak aspect of tellurite fibers make them a very interesting medium to provide broadband Raman amplification since not only different pumps can be combined to flat the gain but also different peaks associated with each pump can be used in the optimization process, which can minimize the number of pumps needed. The attenuation as a function of wavelength can be seen in Fig.3, where a value of $27.6 \mathrm{~dB} / \mathrm{km}$ is found at $1550 \mathrm{~nm}$. Those high values of attenuation prevent this fiber to be used as a gain and transmission medium at the same time. However, its high Raman peak gain allows the use of very short lengths of fiber (few hundreds of meters) in discrete amplifier configurations, minimizing the effects of the high attenuation.

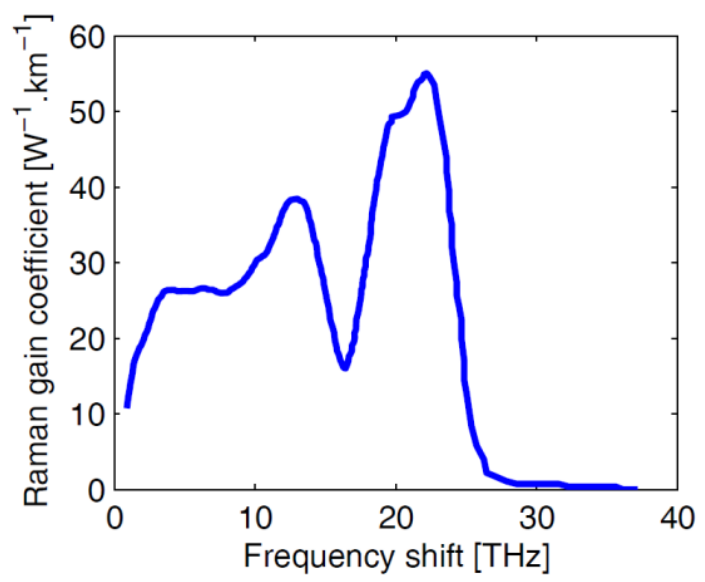

Fig. 2. Raman gain as a function of the pump-signal frequency shift for our tellurite fiber. Obtained from [15].

For the optimization process the pump wavelengths were allowed to vary from 1360 to $1500 \mathrm{~nm}$ and their initial powers from 600 to $800 \mathrm{~mW}$ each in order to assure that we are proposing a low-cost system. The 2-pump system was optimized using an exhaustive search technique based on the one used in [16], which utilizes a very fast simplified analytic model [17] to simulate multi-pump systems allowing the generation of hundred thousands of different configurations within minutes. This fast technique allowed us to generate all possible combinations varying each pump wavelength by $2 \mathrm{~nm}$ and each pump power by $20 \mathrm{~mW}$ within the chosen range, which gives a total of 609961 different cases. In order to assure higher precision for the final results, the best chosen configuration were later re-simulated using an very accurate numerical model, similarly of what was done in [16].

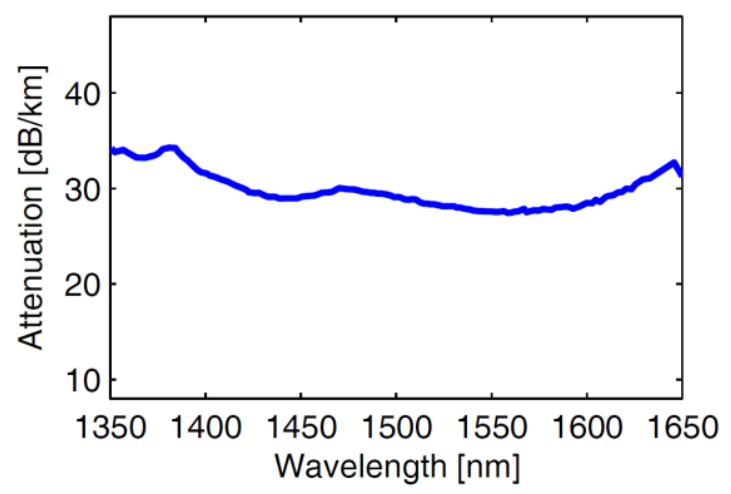

Fig. 3. Attenuation as a function of wavelength for our tellurite fiber. Obtained from [15]. 
The 3-pump systems were optimized using a fast-budget heuristic, which was firstly proposed in [18] that in our optimization process is used to find an initial case solution that is later further reoptimized using an nondominated sorting genetic algorithm which was also described in [18]. Both heuristics simulate the Raman amplification systems using a very precise numerical model, and not an approximated analytic solution. This strategy of optimization used for the 3-pump system was made since the analytic model used for the 2-pump system was not robust enough to produce very accurate solutions when the total pump power increased to values over approximately 1.8 watts.

Finally, the optimization process was used for tellurite fiber lengths varying from 30 to 300 meters in order to find not only the best pump scheme but also the fiber length which allows the optimization process to produce the results with the highest gains and low ripple. Optimization using a standard single-mode telecom fiber (SMF) as the Raman gain medium was also performed under the exactly same pump conditions for longer fiber lengths in order to allow a comparison between both amplification systems. However, it is worth mention that the very reduced lengths used in the discrete configurations based on the tellurite fiber possibly means an advantage when costs and practical implementation are taken into consideration.

\section{RESULTS}

\section{A. Two-pumps system}

For systems using two pumps the highest net gains achieved maintaining the levels of ripple around $2.5 \mathrm{~dB}$ can be seen as a function of the tellurite fiber length in Fig. 4. The highest average gains are around $5.8 \mathrm{~dB}$ and can be achieved for fiber lengths from 90 to $140 \mathrm{~m}$. For longer lengths the high attenuation of the tellurite fiber quickly reduces the achievable gain, preventing its use as an amplifier for lengths over 300 meters. The best optimized system was found to happen when using 100 meters of the gain medium, pump wavelengths of 1398.0 and $1434.0 \mathrm{~nm}$ and pump powers of 800.0 and $720.0 \mathrm{~mW}$ respectively. Its Net gain as a function of wavelength can be seen in Fig. 5.

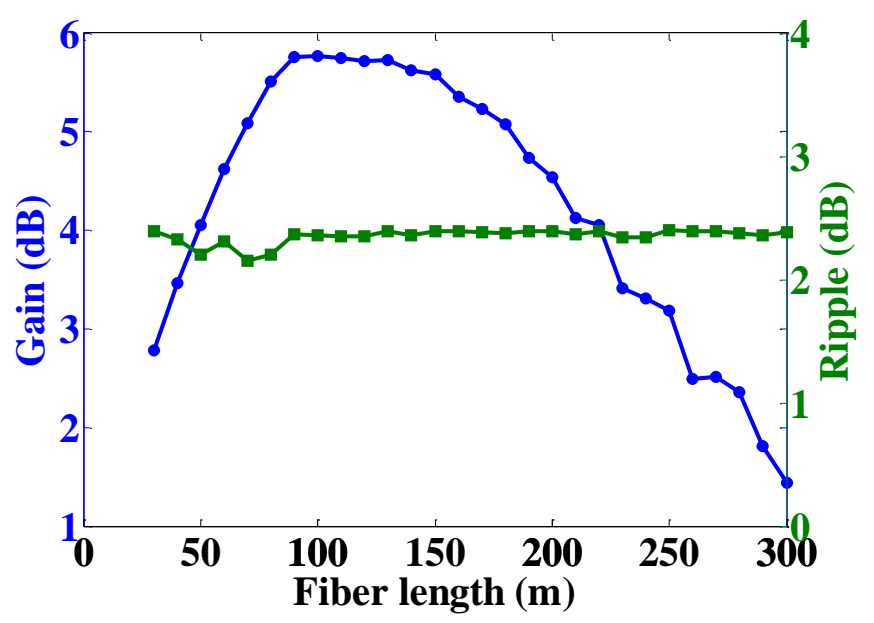

Fig. 4. Average net gain (circle) and ripple (squared dots) as a function of the tellurite fiber length for optimized systems using two pumps. 
A similar optimization was performed using a standard SMF instead of the tellurite fiber as the gain medium and the average gain and ripple values for the optimized systems as a function of the fiber length can be seen in Fig. 6. Slightly higher values of average net gain of up to $6.5 \mathrm{~dB}$ can be achieved for a range of fiber lengths lower than $25 \mathrm{~km}$. For longer distances, the achievable gain falls to lower than $6 \mathrm{~dB}$ and it approaches $4 \mathrm{~dB}$ at lengths around $50 \mathrm{~km}$.

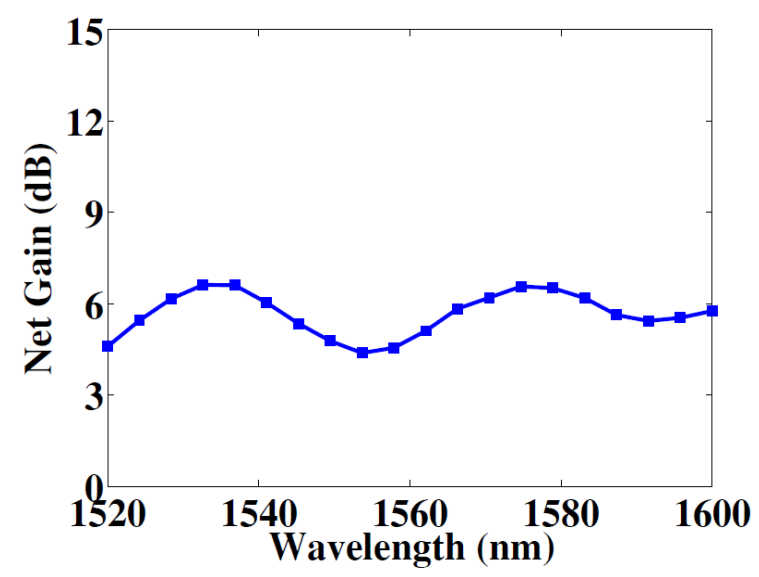

Fig. 5. Net gain as a function of wavelength for the optimized two pump system using $100 \mathrm{~m}$ of tellurite fiber as the gain medium.

The highest average net gain achieved maintaining a ripple lower than $2.5 \mathrm{~dB}$ was found to be 6.76 $\mathrm{dB}$ for $20 \mathrm{~km}$ of SMF, in a system using the pump wavelengths of 1426 and $1476 \mathrm{~nm}$ with pump powers of $800 \mathrm{~mW}$ and $740 \mathrm{~mW}$, respectively. A summary of the best optimization results found for two pump systems in both Silica and Telluride-based fibers is shown in Table I. This result highlights the fact that, depending on the transmission distance, very short discrete amplifier schemes using tellurite fibers as the gain medium and only two pumps with moderate powers can be a simple and low-cost solution to replace or to be used together with distributed Raman amplification in broadband telecommunication systems.

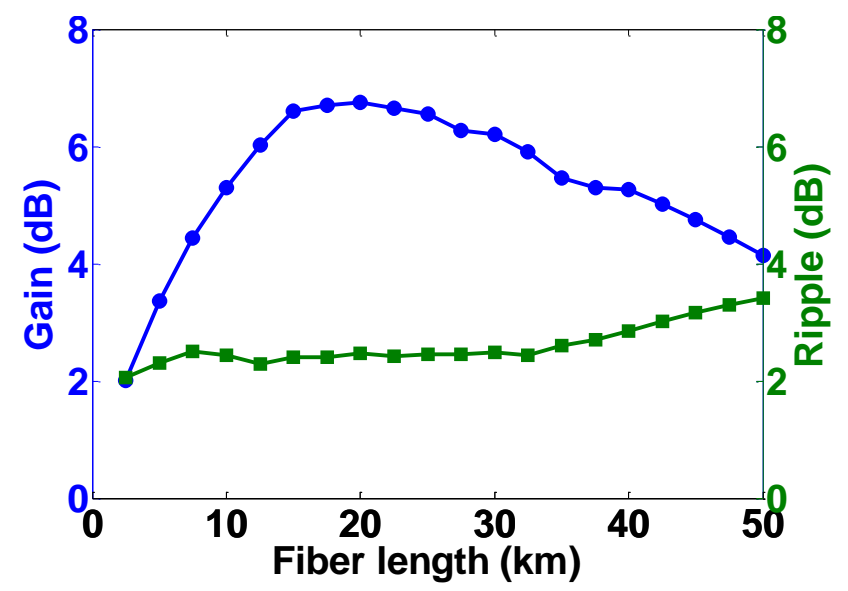

Fig. 6. Average net gain (circle) and ripple (squared dots) as a function of the SMF length for optimized systems using two pumps. 
TABLE I. RESULTS FOR TWO-PUMPS CONFIGURATION

\begin{tabular}{|c|c|c|c|c|c|}
\hline Fiber & $\lambda_{1,2}(\mathbf{n m})$ & $\mathbf{P}_{1,2}(\mathbf{m W})$ & Gain (dB) & Ripple (dB) & Length (m) \\
\hline Silica SMF & 1426.01476 .0 & 800.0740 .0 & 6.76 & 2.47 & 20,000 \\
\hline Tellurite-based fiber & 1398.01434 .0 & 800.0720 .0 & 5.79 & 2.36 & 100 \\
\hline
\end{tabular}

\section{B. Three-pumps system}

The results for average net gain and ripple as a function of the tellurite fiber lengths are shown in Fig. 7. Average gains as high as $10 \mathrm{~dB}$ were achieved for a number of different fiber lengths still presenting ripples around $3 \mathrm{~dB}$. The system with the highest gain that presented a ripple below $3 \mathrm{~dB}$ was found when using $210 \mathrm{~m}$ of tellurite fiber and the pump wavelengths of 1384.7, 1452.6 and $1483.6 \mathrm{~nm}$ with powers of $786.4,782.9$ and $777.4 \mathrm{~mW}$, respectively. Table II summarizes the pump features for silica SMF and tellurite fiber, as well as the gain, ripple and fiber length. The gain as a function of wavelength for this configuration can be seen in Fig. 8 showing an average gain of 9.98 $\mathrm{dB}$ and a ripple of $2.55 \mathrm{~dB}$.

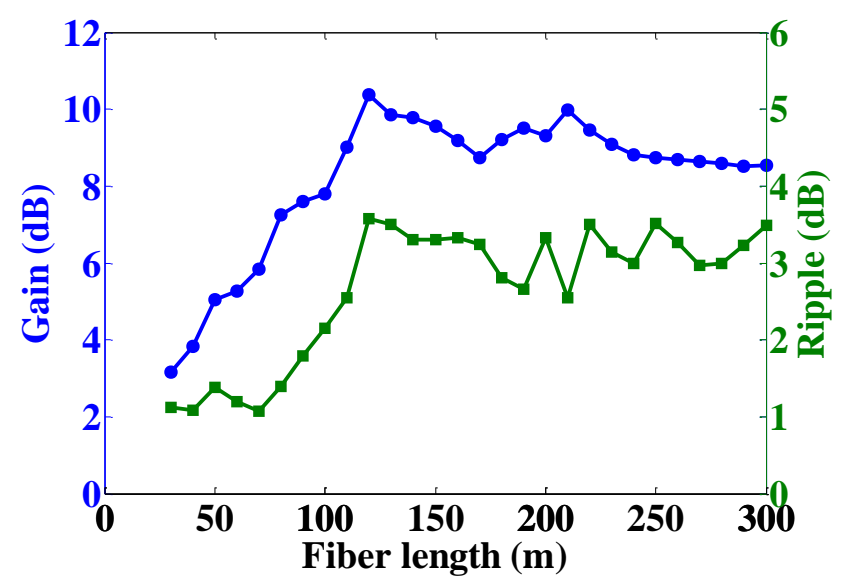

Fig. 7. Average net gain (circle) and ripple (squared dots) as a function of the tellurite fiber length for optimized systems using three pumps.

Once more to drawn a comparison, an optimization of three pump Raman amplification systems were performed using an SMF as the gain medium, and the result of average gain and ripple as a function of the fiber length can be seen in Fig. 9. Average gains near $15 \mathrm{~dB}$ were achieved for all fiber lengths above $50 \mathrm{~km}$ still showing ripples lower than $3 \mathrm{~dB}$. However, for a fiber length of $20 \mathrm{~km}$ the average gain falls to approximately $11 \mathrm{~dB}$, being even smaller for shorter distances.

This fall in the gain for short SMF distances is expected since the low Raman gain of silica fibers do not allow very high average gain amplifiers to be built when the transmission fiber is not long enough. This result again shows that a simple and very short three-pumped tellurite Raman amplification scheme can be used as a discrete amplification module to provide high gains for telecommunication systems which cannot be obtained from distributed amplification alone under certain conditions. The best three-pump optimized results obtained for both fibers are shown in Table II. 


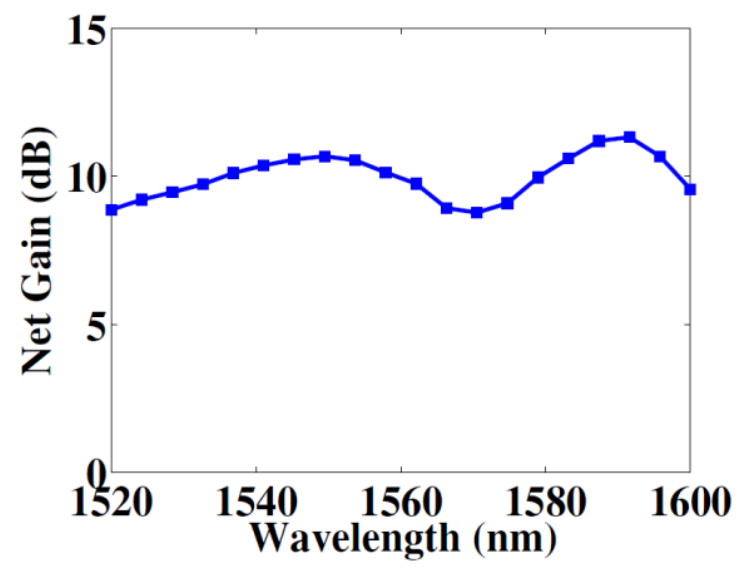

Fig. 8. Net gain as a function of wavelength for the optimized three pump system using $210 \mathrm{~m}$ of tellurite fiber as the gain medium.

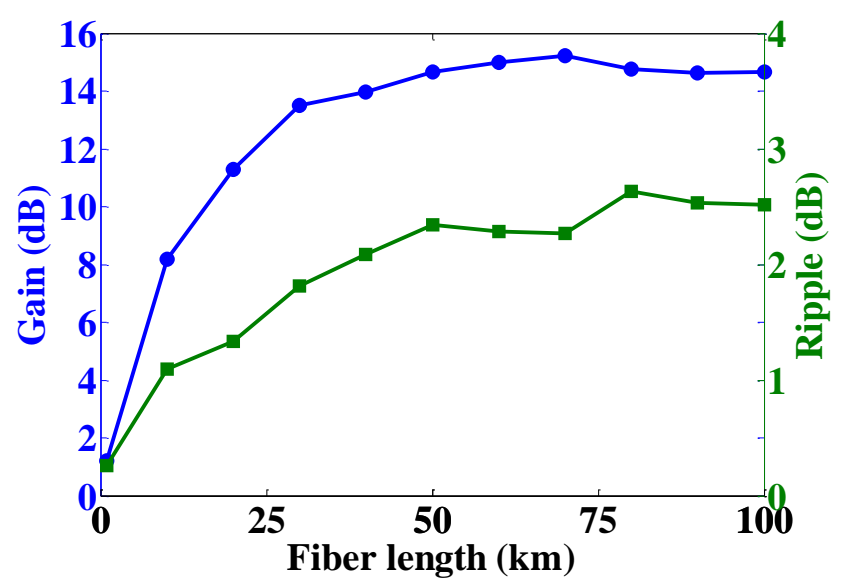

Fig. 9 Average net gain (circle) and ripple (squared dots) as a function of the SMF length for optimized systems using three pumps.

TABLE II. RESULTS FOR THREE-PUMPS CONFIGURATION

\begin{tabular}{|c|c|c|c|c|c|}
\hline Fiber & $\lambda_{1,2,3}(\mathbf{n m})$ & $\mathbf{P}_{1,2,3}(\mathbf{m W})$ & Gain (dB) & Ripple (dB) & Length (m) \\
\hline Silica SMF & 1421.91435 .11476 .4 & 887.9801 .6699 .4 & 15.2 & 2.27 & 70,000 \\
\hline Tellurite-based fiber & 1384.71452 .61483 .6 & 786.4782 .9777 .4 & 9.98 & 2.55 & 210 \\
\hline
\end{tabular}

\section{CONCLUSION}

Here we numerically demonstrate that the high Raman gain present in tellurite-based fibers and its two-peak feature can be exploited to build very short discrete amplification schemes to be used in broadband telecommunication systems. Optimizations were performed not only in terms of pump wavelengths and powers but also analyzing different lengths of the fiber used as the gain medium. Systems with two and three pumps with moderate powers allowed average gains of up to 5.8 and 10 $\mathrm{dB}$, respectively, to be achieved presenting ripples around $2.5 \mathrm{~dB}$ over a bandwidth of $80 \mathrm{~nm}$. A comparison with distributed Raman systems using only SMF was performed highlighting that for 
certain transmission distances the gain achieved from a discrete tellurite fiber, made of a $\mathrm{TeO}_{2}-\mathrm{Bi}_{2} \mathrm{O}_{3}$ $\mathrm{ZnO}-\mathrm{Na}_{2} \mathrm{O}$ (TBZN) glass, with only a few hundreds of meters as the gain medium can be higher.

\section{ACKNOWLEDGMENT}

The authors would like to thank and acknowledge funding received from Conselho Nacional de Desenvolvimento Científico e Tecnológico (CNPQ), Coordenação de Aperfeiçoamento de Pessoal de Ensino Superior (CAPES), Fundação de Amparo a Pesquisa do Espírito Santo (FAPES) and Petróleo Brasileiro S.A. (Petrobras) for the development of this research.

\section{REFERENCES}

[1] A. Lin, A. Zhang, E. J. Bushong, and J. Toulouse, "Solid-core tellurite glass fiber for infrared and nonlinear applications," Opt. Express, vol. 17, pp. 16716-16721, 2009.

[2] N. Manikandan, A. Ryasnyanskiy, J. Toulouse, "Thermal and optical properties of $\mathrm{TeO}_{2}-\mathrm{ZnO}-\mathrm{BaO}$ glasses," Journal of Non-Crystalline Solids, vol. 358, pp. 947-951, 2012.

[3] C. Strutynski, P. Froidevaux, F. Désévédavy, J. C. Jules, G. Gadret, A. Bendahmane, K. Tarnowski, B. Kibler, and F. Smektala, "Tailoring supercontinuum generation beyond $2 \mu \mathrm{m}$ in step-index tellurite fibers," Opt. Lett., vol. 42, 247250, 2017.

[4] T. Cheng, K. Asano, Z. Duan, T. H. Tuan, W. Gao, D. Deng, T. Suzuki, Y. Ohishi, "Design and optimization of tellurite hybrid microstructured optical fiber with high nonlinearity and low flattened chromatic dispersion for optical parametric amplification," Optics Communications, vol. 318, pp. 105-111, 2012.

[5] G. Zhu, L. Geng, X. Zhu, L. Li, Q. Chen, R. A. Norwood, T. Manzur, and N. Peyghambarian, "Towards ten-watt-level 3-5 $\mu \mathrm{m}$ Raman lasers using tellurite fiber," Opt. Express, vol. 23, pp. 7559-7573, 2015.

[6] D. Manzani, Y. Ledemi, I. Skripachev, Y. Messaddeq, S. J. L. Ribeiro, R. E. P. de Oliveira, and C. J. S. de Matos, " $\mathrm{Yb}^{3+}, \mathrm{Tm}^{3+}$ and $\mathrm{Ho}^{3+}$ triply-doped tellurite core-cladding optical fiber for white light generation" Opt. Mater. Express, vol. 1, pp. 1515-1526, 2011.

[7] R. Stegeman, L. Jankovic, H. Kim, C. Rivero, G. Stegeman, K. Richardson, P. Delfyett, Y. Guo, A. Schulte, and T. Cardinal, "Tellurite glasses with peak absolute Raman gain coefficients up to 30 times that of fused silica," Opt. Lett., vol. 28, pp. 1126-1128, 2003.

[8] T. Cheng, Tong Hoang Tuan, Xiaojie Xue, Dinghuan Deng, Takenobu Suzuki, Yasutake Ohishi,"Optical solitons and supercontinuum generation in a tellurite microstructured optical fiber," Optics Communications, vol. 369, pp.159-163, 2016.

[9] M. Boivin, M. El-Amraoui, Y. Ledemi, S. Morency, R. Vallée, and Y. Messaddeq, "Germanate-tellurite composite fibers with a high-contrast step-index design for nonlinear applications," Opt. Mater. Express, vol. 4, pp. 1740-1746, 2014.

[10] J. Lia, X. Xiaob, S. Gua, Y. Xub, Z. Zhoub, H. Guo, "Preparation and optical properties of TeO2-BaO-ZnO-ZnF2 fluoro-tellurite glass for mid-infrared fiber Raman laser applications," Optical Materials, vol. 66, pp. 567-572, 2017.

[11] T. Cheng, W. Gao, X. Xue, T. Suzuki, and Y. Ohishi, "Experimental investigation of multiple Raman peak properties in a hundred-meter tellurite fiber," Opt. Mater. Express, vol. 6, pp. 3438-3445, 2016.

[12] G. Qin, M. Liao, T. Suzuki, A. Mori, and Y. Ohishi, "Widely tunable ring-cavity tellurite fiber Raman laser," Opt. Lett., vol. 33, pp. 2014-2016, 2008.

[13] G. Qin, R. Jose, and Y. Ohishi, "Design of Ultimate Gain-Flattened O-, E-, and S+ C+ L Ultrabroadband Fiber Amplifiers Using a New Fiber Raman Gain Medium," J. Lightwave Technol., vol. 25, pp. 2727-2738, 2007.

[14] H. Masuda, A. Mori, K. Shikano, and M. Shimizu, "Design and Spectral Characteristics of Gain-Flattened TelluriteBased Fiber Raman Amplifiers," J. Lightwave Technol., vol. 24, pp. 504-515, 2006.

[15] A. Mori, H. Masuda, K. Shikano and M. Shimizu, "Ultra-Wideband Tellurite-Based Fiber Raman Amplifier," J. Lightwave Technol., vol. 21, pp. 1300-1306, 2003.

[16] C. E. S. Castellani, S. P. N. Cani, M. E. V. Segatto, M. J. Pontes, and M. A. Romero, "Design methodology for multipumped discrete Raman amplifiers: case-study employing photonic crystal fibers," Opt. Express, vol. 17, pp. 1412114131, 2009.

[17] S. P. N. Cani, L. C. Calmon, M. J. Pontes, M. R. N. Ribeiro, M. E. V. Segatto, and A. V. T. Cartaxo, "An Analytical Approximated Solution for the Gain of Broadband Raman Amplifiers With Multiple Counter-Pumps," J. Lightwave Technol., vol. 27, pp. 944-951, 2009.

[18] H. R. O. Rocha, C. E. S. Castellani, J. A. L. Silva, M. J. Pontes, M. E. V. Segatto, "Fast optimization of multipump raman amplifiers based on a simplified wavelength and power budget heuristic," Opt. Eng., vol. 54, pp. 015105, 2015. 